\title{
High-Precision Photometry with Small Telescopes on the Moon
}

\author{
Michael Zeilik \\ Institute for Astrophysics, The University of New Mexico
}

\begin{abstract}
I explore the scientific gains that can be achieved by siting small ( 1 to 2 meter), special-purpose telescopes on the moon with the goal of high-precision observations.
\end{abstract}

\section{Introduction}

Robust small telescopes ( 1 to 2 meters) will likely pioneer astronomy on the moon. These light-weight, robotic instruments could even be soft-landed to the moon prior to the establishment of a lunar base. Each could be designed for a specialized task, such as imaging with detectors for limited ranges of the spectrum selected for specific scientific goals. Lunar APTs (LAPTs; Zeilik, 1989) would function much like those on the earth for interleaved programs of target objects. Initial instruments could operate as Lunar Transit Telescopes (LTTs). A LTT provides an unbiased, deep sample of a designated strip of sky over time. In either mode, we can imagine a network of lunar telescopes to cover the full sky (especially the southern) at a range of wavelengths to achieve a wide variety of scientific goals unattainable on earth.

The moon is by far the best site in the inner solar system for the next generation of high-performance telescopes. The main advantages of the moon relate to the fact that it is a stable, slow-rotating space platform (near the earth) on which large (and small!) structures can be built in an environment with no atmosphere. Lunarbased telescopes can make powerful utility of the lunar advantages for astronomy: no atmospheric opacity, so that all regions of the spectrum are open to view; no atmospheric seeing, so that the resolution is limited by the optics; no weather, so that long strings of precise, time-serial data are straight-forward to obtain; and no sky backgrounds to limit $\mathrm{S} / \mathrm{N}$, so even small telescopes can detect faint objects with relatively short integration times.

\section{CCDs and Small Telescopes on the Moon}

The Lunar advantages easily optimize the use of CCDs on small telescopes on the moon. If we apply the S/N CCD equation of Newberry (1991), we quickly see that: (1) we optimize object counts (100\% transparent and constant with no air!); (2) we decrease background counts (lower sky brightness, less variation); (3) we can reduce number of pixels covered by object (no seeing, diffraction limited, match pixel 
size to optics); and (4) we can tailor filters to detectors and standard photometric systems as well as satisfying the sampling theorem (no second order extinction!). In conclusion: Low sky background, diffraction-limited performance optimizes the use of CCDs on small lunar telescopes to make them work effectively as large, ground-based telescopes.

In addition, small telescopes have the practical advantages that they have a low mass; are relatively inexpensive; rely on proven technology; are designed to be simple, robotic, and robust; can be soft landed; function as precursors to giant lunar telescopes to test the working conditions in the lunar environment with cosmic rays, meteoroids, large thermal variations between day and night, and charged surface dust.

\section{Lunar Automatic Photometric Telescopes (LAPTs)}

As a simple case, consider a 1 to $2-\mathrm{m}, \mathrm{f} / 2$ primary, $\mathrm{f} / 5$ to $\mathrm{f} / 10$ Cassegrain telescope. It will have a resolving power of 0.1 arcsec at $600 \mathrm{~nm}$; a mass of some $1000 \mathrm{~kg}$; and be passively cooled. As a pointed telescope (accuracy $<10 \mathrm{arcsec}$ ), it will have altazimuth mount, so that it is latitude independent. The detector would be a mosaic of CCDs (about 2000 by 2000 pixels; a 10 micron pixel size) at a focal plane, a minimum of 2 colors (with a dichroic beam splitter), and a field of view of some 10 by 10 arcmin. The CCDs would provide target acquisition: an exposure of a few seconds detects stars down 15 th mag at a $\mathrm{S} / \mathrm{N}>3$ (about 10 stars per frame). We would provide software masks for target identification with artificial intelligence algorithms for rapid confirmation. Once the right field is established, a $500 \mathrm{~s}$ exposure gives $\mathrm{S} / \mathrm{N} \approx 2000$ $(0.05 \%)$ for a $V=15$ point source of $S / N \approx 200$ for $V=20$. We can rapidly attain high-precision, time-serial, continuous data.

What kinds of science could LAPTs achieve? One major target would be variable sources with multiple periodic structure, such as chromospherically active binary start systems, quasars, and AGNs. We could obtain performance impossible on the earth by collecting time-serial, continuous data of high-precision ( $\mathrm{S} / \mathrm{N}=100$ minimum) on selected targets. Such long data strings will allow us to extract reliably all the periods displayed by the target objects.

We can go beyond a single telescope and imagine a LAPTs network with eight telescopes, four in each hemisphere set at a 90 degree spread in longitude. A network would increase continuity of coverage, the number objects observed, and the wavelength regime for simultaneous data. Of course, it would be coordinated with any earth-based networks to maximize the scientific return.

\section{Lunar Transit Telescopes (LTTs)}

An even simpler design is that of a Lunar Transit Telescope (McGraw, 1992), which uses the moon's rotation as a slow and stable telescope drive. This instrument points at the meridian; the integration time is simply the time of an object's transit of the $\mathrm{d}$ detector at the focal plane. 
Consider a $2-\mathrm{m}$ telescope (mass about $1000 \mathrm{~kg}$ ), with a FOV of about 2 degrees, and all-reflective optics. With a pin-joint structure, it would undergo passive thermal self compensation and never need to be focused. The detector would be a mosaic of UVOIR CCDs, with 0.1 arcsec pixels, and 5 bandpasses: 0.1 to 2 microns. The lunar sidereal rate gives 6.6-min integration on 2048 pixel array, so we expect limiting magnitudes of +27 at $\mathrm{V},+25$ at $\mathrm{H}$, and +24.5 at $\mathrm{K}$ for a $\mathrm{S} / \mathrm{N}=10$. As the sky slides by, an LTT will survey about 700 square degrees each lunar day. It will provide an unbiased, "deep" sample of homogeneous, high-precision, multicolor photometry.

An LLT will provide a vast amount of data - a strip search of the universe that will reveal millions of objects, some at physical extremes. It will be serendipity at its astronomical best, as the sample will be statistically complete down to the limiting magnitudes. For instance, we can study the morphology of distant galaxies and with multicolor imaging, provide evidence for a firm scenario of galaxian evolution. At this range of lunar astronomy, we will be constrained more by our imagination than by our data.

This work was funded in part by National Science Foundation Grant AST-8903174.

\section{References:}

Newberry, M. V., 1991, Pub. Astron. Soc. Pac., 103.122.

McGraw, J. T., 1992, in AIP Conference Proceeding 207, Astrophysics on the Moon, ed. M. J. Mumma and H. J. Smith, p. 433.

Zeilik, M., 1989, "Remote Access Lunar Photometric Telescopes" in Remote Access Automatic Telescopes, ed. D. S. Hayes and R. M. Genet (Fairborn Pres), pp. 289-298.

\section{Discussion}

A. T. Young: I question your assumption that a telescope on the Moon is cheap, even if small. I was involved in a $1 \mathrm{~cm}$ telescope on Mars (Viking Lander camera), which cost about 16 million - before launch costs. Also, you have little dark time on the Moon. The Earth in the lunar sky is about 4 magnitudes brighter than the Moon in the terrestial sky.

Zeilik: Any space mission for astronomy is expensive. In relative terms, a low-mass payload is cheaper. The phases of the Earth do make a bright source, especially at full Earth. But the Moon has no atmosphere to scatter the light, and the problem is less severe than in low-Earth orbit.

S.B. Howell: The radiation event problem will cause real damage to the CCD. In timeseries mode, these events will cause the loss of photometric information for a few frames, but most of the series will be useable. 
Zeilik: I hope that a cosmic-ray hit will not affect more than 1 or 2 pixels and can be removed in processing. However, with a flux $\sim 100$ times that on the earth, actual damage may well result.

R.F. Garrison: One disadvantage you didn't list, with respect to geosynchronous orbits, is that only one half of the sky is available at any given time.

Zeilik: Yes. That's the reason I proposed a network on the Moon for all-sky coverage.

E. Budding: I think that, if you want to see real data from this kind of remotely operated device, (including also the SALUTE-type instrument) before retirement, you should be pushing hard for the terrestrial-precursor version.

Zeilik: The CCD Transit Instrument (CTI) is an already operational precursor to the Lunar Transit Telescope.

D.H.P. Jones: I understand there are some deep craters at the poles of the Moon which are in continuous darkness.

Zeilik: Such craters would work as excellent sites for completely-cooled infrared telescopes. 


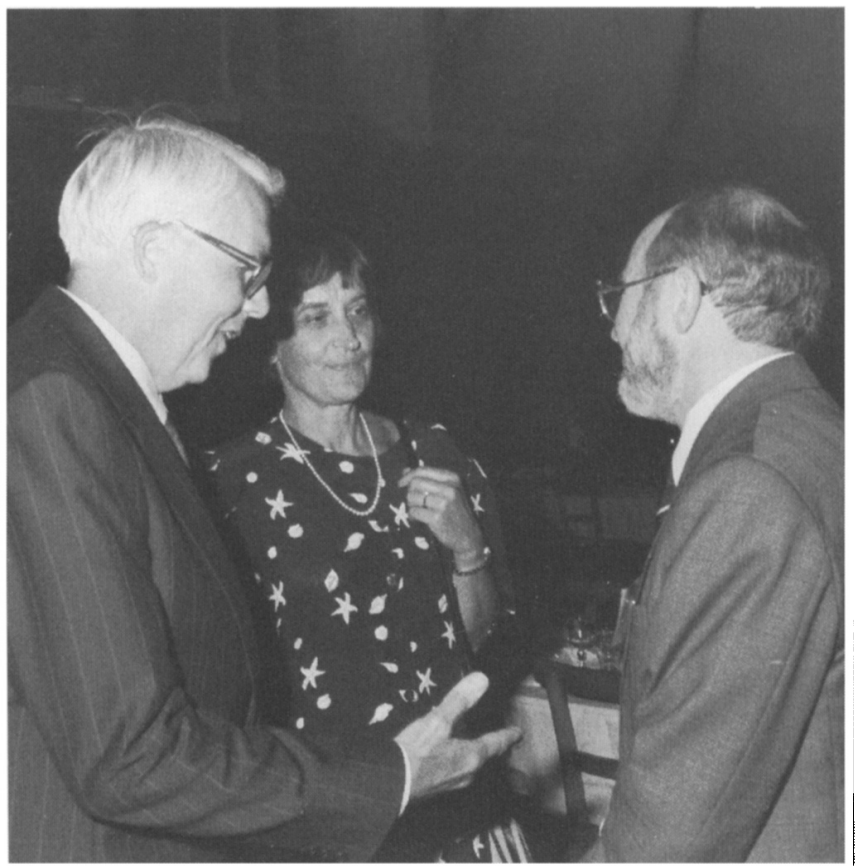

Tarmo and Silvi Oja say good-bye to Ian Elliott 


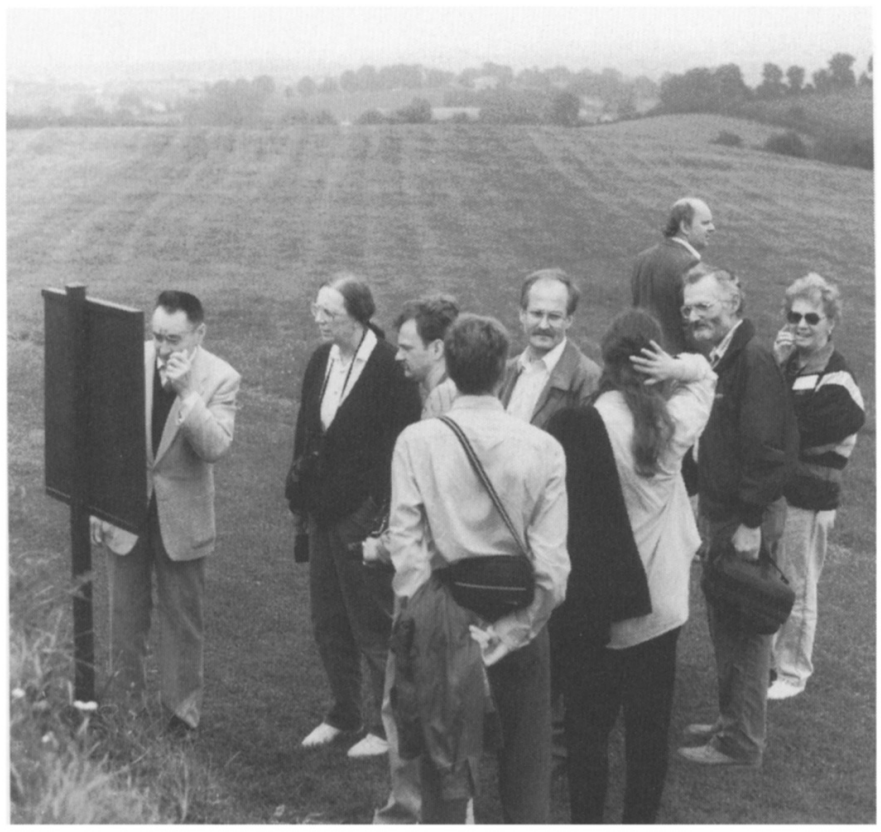

A group at the prehistoric site of Navan Fort near Armagh 